\title{
Lethal Kniest-like dysplasia
}

INSERM

\section{Source}

INSERM. (1999). Orphanet: an online rare disease and orphan drug data base. Lethal Kniest-like dysplasia. ORPHA:2347

A rare, lethal, congenital, chondrodysplasia disorder characterized by dumbbell-shaped long bones with markedly shortened diaphyses and metaphyseal irregularities associated with a "Swiss cheese" appearance of the cartilage matrix, as well as distinctive changes in the growth plate and resting cartilage, resulting in death in the neonatal period. There have been no further descriptions in the literature since 1983. 